\title{
Target detection and localization in visual search: A dual systems perspective
}

\author{
GENIVA LIU \\ University of British Columbia, Vancouver, British Columbia \\ CHRISTOPHER G. HEALEY \\ North Carolina State University, Raleigh, North Carolina \\ and \\ JAMES T. ENNS \\ University of British Columbia, Vancouver, British Columbia
}

\begin{abstract}
The dual visual systems framework (Milner \& Goodale, 1995) was used to explore target detection and localization in visual search. Observers searched for a small patch of tilted bars against a dense background of upright bars. Target detection was performed along with two different localization tasks: direct pointing, designed to engage the dorsal stream, and indirect pointing, designed to engage the ventral stream. The results indicated that (1) target detection was influenced more by orientation differences in 3-D space than by 2-D pictorial differences, (2) target localization was more accurate for direct than for indirect pointing, and (3) there were performance costs for indirect localization when it followed target detection, but not for direct localization. This is consistent with direct localization's having greater dependence on the dorsal visual system than either target detection or indirect localization.
\end{abstract}

Visually guided actions typically require both the correct detection of an object and its accurate localization. In order to reach for a cup or pick up a pencil, we must first detect relevant object features and then direct our actions appropriately toward the object. At least this is how many theories of visually guided action have conceptualized the problem in the past. Although there has been some debate about whether object features are "bound" to one another in a mental representation before the bundle of features that form an object has been localized in space (Treisman \& Gelade, 1980) or only thereafter (Julesz, 1984), it has simply been taken for granted that object detection and localization are functionally linked to each other.

However, a new way of conceptualizing the relationship between object detection and localization has recently emerged. It is premised on the existence of two separate visual pathways in the brain: the ventral stream and the dorsal stream (Goodale \& Milner, 1992; Milner $\&$ Goodale, 1995). The ventral stream is specialized for object identification and the conscious aspects of perception, whereas the dorsal stream is specialized for the online and largely unconscious control of action. Although each pathway supports the detection and localization of objects in some sense, they do so in very dif-

This research was supported by the Natural Sciences and Engineering Council of Canada and by National Science Foundation Grant ACI0083421. Correspondence should be addressed to G. Liu, Department of Psychology, 2136 West Mall, University of British Columbia, Vancouver, BC, V6T 1Z4 Canada (e-mail: geniva@interchange.ubc.ca). ferent ways and for different purposes. For instance, the detection of an object in the ventral stream is associated with conscious experience and mental representations aimed at object identification (i.e., emphasizing intrinsic object properties). Object localization in this pathway is therefore aimed at representing the relationship among objects in the environment. In contrast, the detection of an object in the dorsal stream is geared toward representing its action-oriented properties, such as its size and orientation with respect to the viewer's reach and grasp.

Of course, even from the perspective of this dual systems theory, in a healthy human these pathways must work together to create coherence in both perceptual experience and action (Goodale \& Milner, 1992; Milner \& Goodale, 1995). But the typically smooth coordination of these systems need not detract from the reality that there are two independent streams of processing that can be decoupled through selective brain damage or by the design of laboratory tasks that differentially emphasize their separate functions. In the present study, we explored the possibility of a psychophysical decoupling in the realm of visual search.

\section{Target Detection and Localization in Visual Search}

The strongest influence on our understanding of visual search over the past 20 years has come from feature integration theory (Treisman \& Gelade, 1980; Treisman \& Sato, 1990). This theory proposes that efficient target detection occurs prior to target localization and that it can even occur independently of localization if the search 
task does not require a localization response. This prediction stems from the proposal that images are analyzed by separate feature maps in the brain (e.g., maps of orientation, color, and motion), which are independent of a master map of locations. Therefore, if a search task requires only the detection of a target on the basis of a difference in one of these features, then detection can occur with no need to access the target's location.

The theory predicts that location information accompanies detection when one of two additional demands is included in the search task. First, if the response made to the target has a spatial component (e.g., Is the target is on the left or on the right?), then the master map must be consulted. Second, if the target cannot be defined on the basis of a single feature dimension (e.g., Is there a vertical red bar among vertical green bars and horizontal red bars?), then target localization will be required. This is because it is only at the level of the master map that separate features of an object are "conjoined"-a process that is attention limited and can be completed only for one object at a time.

Support for this relationship between target detection and target localization was reported by Treisman and Gelade (1980), who showed that targets defined by simple features could be detected very accurately even when there were large inaccuracies in their localization. In comparison, the detection accuracy of targets defined by feature conjunctions was much more similar to the localization accuracy of these conjunction-defined targets.

Feature integration theory has not gone undisputed. In particular, texton theory (Julesz, 1984; Sagi \& Julesz, 1985a) predicts an opposite relationship between object detection and localization. According to texton theory, target detection occurs only after an initial stage of processing in which the visual image has been analyzed for spatially localized discontinuities in a small set of elementary shape features (e.g., elongated blobs, blob endings, and blob intersections). Discontinuity localization of these features is implemented as a parallel process and is therefore not attention limited. On the other hand, detection of these features requires serial inspection and is therefore limited by attention. Because of this proposed division of labor between the parallel processes of discontinuity localization and the serial processes of target identification, the prediction is that targets in a visual search task can be located before their feature identity can be detected.

In support of this theory, Sagi and Julesz (1985a, $1985 b$ ) reported a dissociation between performance in the detection and discrimination of orientation-defined targets. Specifically, target detection yielded no differences in search time as a function of target number, but discrimination search times increased as target number increased. In a separate experiment, detection performance was compared to a localization task in which observers had to indicate the configuration of targets, which required a positional judgment regarding target locations. The results of this experiment yielded over- lapping psychometric curves for the two tasks. On the basis of these results, it would seem that spatial discontinuities can be detected and localized in parallel, but that detection of the target on the basis of its identity can only be done serially. However, even this interpretation has recently been challenged on the grounds that the detection task was a single task (detect local spatial discontinuities), whereas the discrimination task involved two perceptual steps (detect discontinuities and then detect feature differences) and, therefore, dual-task interference was causing impairment in the discrimination task (Di Lollo, Kawahara, Zuvic, \& Visser, 2001).

Studies of the opposing predictions of feature integration theory and texton theory have yielded mixed results. For example, Green (1992) reported that if an orientationdefined target is correctly detected, then it can also be localized to the extent that observers can accurately indicate the side of the visual field on which the target was presented. In a further refinement of this idea, Atkinson and Braddick (1989) reported that for masked displays of oriented lines, targets defined by an orientation difference could be localized to a high degree of spatial resolution for relatively longer display durations, but could only be localized to a coarser spatial resolution when the same targets appeared in shorter displays. In summary, neither texton theory nor feature integration theory provides a comprehensive framework for understanding the relationship between target detection and localization.

\section{Dual Systems Theory}

Inspired by findings in neuropsychology and neuroscience, theories of two visual systems provide a different way of thinking about target detection and localization. In these theories, it is proposed that the visual system is composed of separate pathways for object identification and localization. In early versions, it was speculated that these systems might diverge in the optic tract between the retina and the visual cortex, with the location pathway consisting of the tectopulvinar route through the superior colliculus and the identification pathway consisting of the geniculostriate route through the lateral geniculate nucleus (Held, 1970; Schneider, 1969; Trevarthen, 1968). More recently, the cortical visual system has itself been characterized as consisting of two pathways: a ventral stream specialized for object perception and a dorsal stream specialized for spatial vision. In the macaque cortex, these streams have been traced anatomically (Ungerleider \& Mishkin, 1982; Van Essen \& DeYoe, 1995). Both streams begin in the primary visual cortex (V1), with the the ventral stream projecting to the inferotemporal cortex and the dorsal stream projecting to the posterior parietal cortex. In humans, brain-imaging data on healthy participants as well as data on neuropsychological conditions in brain-damaged individuals are consistent with this general scheme (Haxby et al., 1991; Tootell, Dale, Sereno, \& Malach, 1996).

The early characterization of the two systems referred to "what" (ventral) and "where" (dorsal) streams (Unger- 
leider \& Haxby, 1994; Ungerleider \& Mishkin, 1982). Goodale and Milner (1992; Milner \& Goodale, 1995) argued that the label of "where" should be replaced with that of "how" in order to emphasize functional differences between the two systems. In their view, the ventral stream analyzes information relevant to conscious perception of objects (e.g., categorization, recognition, and identification), whereas the dorsal stream analyzes information needed to guide actions directed toward objects (e.g., pointing, reaching, and grasping). This action-guidance function of the dorsal stream is generally completed more rapidly than the functions of the ventral stream, and its results are less available to consciousness.

There is considerable neuropsychological evidence to support the independence of these two streams in humans. For example, patients with the clinical syndrome of optic ataxia (Bálint, 1909) typically have damage in the superior portion of the posterior parietal cortex and show impaired dorsal stream function with spared ventral stream function. They may be unable to use vision to reach for and grasp objects that they are nonetheless able to recognize and describe verbally (Goodale, Milner, Jakobson, \& Carey, 1991; Perenin \& Vighetto, 1988). Conversely, other patients exhibit impaired ventral stream function with spared dorsal stream function. One of the best known examples is D.F., who sustained extensive damage to the ventrolateral regions of her occipital lobe, leaving her with severe visual form agnosia (Milner et al., 1991). Although D.F. is unable to identify or describe characteristics of visually presented objects (e.g., the orientation of a slot in a mailbox), she is able to use these characteristics to accurately direct actions toward the same objects (e.g., she can post a letter in the slot).

Dual systems theory, therefore, raises questions about object detection and localization that are different than those raised by the earlier theories premised on a single system. For feature integration theory and texton theory, the main questions were whether either of these functions could be performed using parallel processes and whether one function was accomplished first, thereby influencing the other. From the perspective of the dual systems theory, a more important question is whether these functions can sometimes be accomplished independently. In some sense, both detection and localization must be performed by each visual stream (Milner \& Goodale, 1995). However, given the way the two systems are specialized, there is a possibility that their independence could be demonstrated if object detection required a report of conscious perceptual experience and object localization required direct and rapid action to the object's location.

A related possibility raised by the dual systems theory is that each system has its own pool of attentional resources. When an observer must coordinate responses to two different questions or tasks concerning the same visual display, there are typically performance costs in comparison with when either of these tasks is performed alone (see, e.g., Braun \& Sagi, 1990; Di Lollo et al.,
2001). The inference is that both tasks require access to the same cognitive resources, leading to a performance decrement in at least one of the tasks (Navon \& Gopher, 1979; D. A. Norman \& Bobrow, 1975, 1976; Wickens, 1984). But if there are separate resources for each stream, then some aspects of object detection could be performed concurrently with some aspects of object localization, provided that these functions are each supported by different streams. Alternatively, to the extent that the demands of object detection and localization are supported by processes in the same stream, then efficient performance of one task should interfere with the concurrent performance of the other. To date, there has been little research devoted to exploring this idea of separate resources (e.g., Castiello, Paulignan, \& Jeannerod, 1991; Ho, 1998; J. Norman, 2002).

\section{Scope of the Present Study}

In this study, the dual systems framework was used to examine target detection and localization in visual search. This was accomplished by combining the traditional requirements of target detection (i.e., Is a target present or absent in the display?) with two different target localization tasks. In one task, the participants indicated target location by pressing a key in a spatial array that corresponded to the display locations. We refer to this localization response as indirect action, because a conscious decision must be made to indicate the target location with a keypress to a spatially corresponding, but different, location. In the second task, the participants indicated target location by pointing directly to the screen location before making the spatially mapped keypress. We call this direct action, because it can be made, in principle, using the unconscious processes of the dorsal stream to guide the finger-pointing action.

Because the dorsal stream is specialized to act on information in 3-D space and because the ventral and dorsal streams are each sensitive to object orientation (Milner et al., 1991; Perenin \& Vighetto, 1988), we designed visual search displays depicting a 3-D textured surface of "grass" (orientation-defined targets and distractors) receding into the distance. An example of one of these displays is shown in Figure 1A. Each blade of "grass" was a short line segment that was "standing up" on a flat surface. The target consisted of a small patch of lines that was tilted by a variable number of degrees, creating a range of target difficulty from very easy to very difficult.

As a first step, we conducted an experiment to compare search for orientation-defined targets in 2-D and 3-D arrays. This was important to establish that the participants were sensitive to the 3-D information depicted in the displays and were actually using this information to guide their responses.

The study then focused on two main questions. First, is there a difference in localization accuracy when participants engage in direct versus indirect actions to indicate target location? To the extent that direct action relies on unconscious and short-lived dorsal-stream spatial information that is not available to the ventral stream, we 

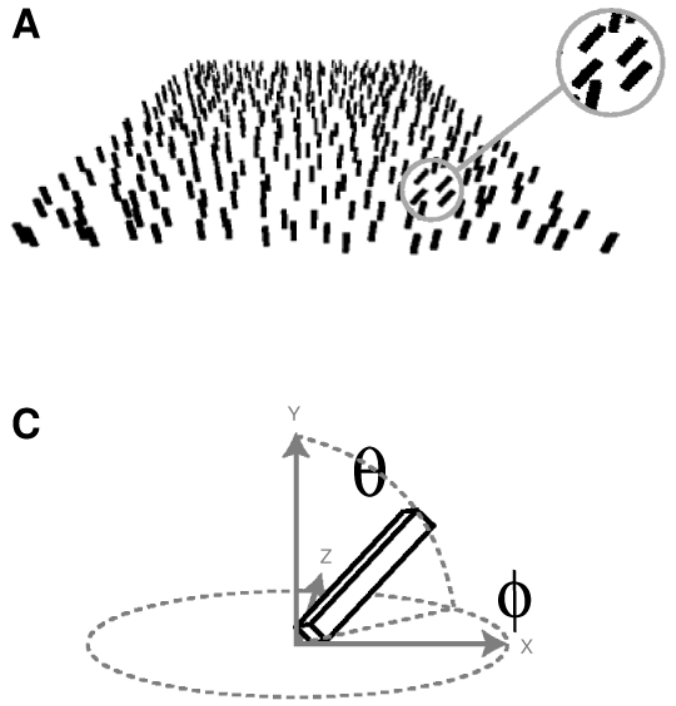

B

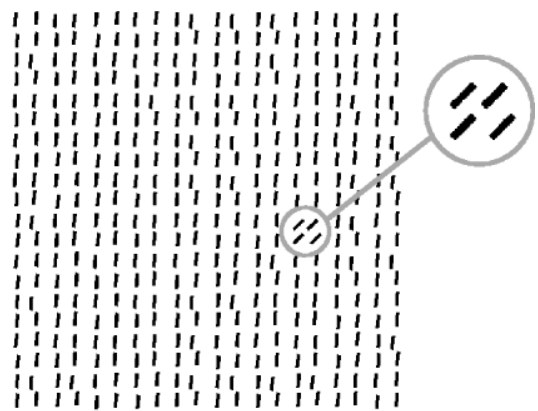

Figure 1. (A) Example of a 3-D search array with target present. For purposes of illustration, the target region is circled and enlarged in the inset. In this example, the target differs from the background elements by $\theta=25^{\circ}$ and $\phi=-15^{\circ}$. Considered only as angular deviation in the picture plane, this corresponds to 2-D tilt $=34^{\circ}$. $(B)$ Example of a 2-D search array with target present. The target region is circled and enlarged in the inset. (C) Components of depicted 3-D orientation. $\theta$ describes angular deviations from the vertical $y$-axis in 3-D, and $\phi$ describes spin around the $y$-axis.

would expect to see greater accuracy in the direct-action condition.

Second, are there separate attentional resources for target detection and target localization? Dual systems theory raises the possibility that ventral and dorsal streams each have their own resources for perceptual processing. If this is the case, we would expect little or no interference when the two tasks draw on different streams. To test this possibility, we compared performance in single-task conditions (i.e., detection alone, indirect localization alone, and direct localization alone) with dual-task conditions (i.e., detection paired with indirect localization and detection paired with direct localization).

\section{EXPERIMENT 1 \\ Visual Search for Orientation-Defined Targets in 3-D Textures}

A necessary first step was to ensure that the participants were sensitive to the depicted 3-D relations in our search displays. At the outset, we did not know whether there were important differences between orientation sensitivity in 2-D and 3-D displays, and we did not know which 3-D display parameters had the greatest influence on visual search. Indeed, one possibility is that sensitivity to orientation in 3-D search displays is based simply on the 2-D orientation differences that can be recorded in the picture plane. To address these issues, we conducted a preliminary experiment in which searches in 2-D and 3-D displays were compared.

There has been some research in which 2-D and 3-D sensitivity was compared using search displays that were more sparsely arrayed than those currently of interest (Enns \& Rensink, 1990a). It showed that visual search for drawings of simple 3-D blocks could be performed efficiently when target blocks and distractor blocks had a $90^{\circ}$ difference in 3-D orientation. This held true even when there were no associated target-distractor differences in 2-D orientation among the elements (lines and shapes) used to depict the 3-D blocks. Direct comparisons of visual search on the basis of 2-D versus 3-D orientation differences indicated that orientation sensitivity was similar in the two domains (Enns \& Rensink, 1990b). However, these findings may have limited generality to the present densely textured arrays of "grass," because they involved relatively large objects $\left(1^{\circ}\right.$ of visual angle or more), sparse displays (no more than 15 objects in a display), and only maximum differences in orientation $\left(90^{\circ}\right)$. Another study revealed that visual search was based in part on the apparent 3-D size of cylinders drawn on a texture gradient (Aks \& Enns, 1996). This suggests that early visual processes employ mechanisms of size constancy, but, again, there are questions about whether this outcome will generalize to the present dense texture displays.

\section{Method}

Participants. Fifty undergraduate students (36 females, 14 males; mean age $=20.8$ years) participated in exchange for course credit. All reported normal or corrected-to-normal vision. Twenty students participated in the 2-D condition (10 students each in the $0^{\circ}$ and $90^{\circ}$ cardinality groups; see the Stimuli and Design section for details). Thirty participated in the 3-D condition. A larger number of students was tested in the 3-D condition in order to increase the statistical power for the larger number of experimental factors analyzed in this condition. 
Stimuli and Design. The participants were seated in a well-lit room viewing a 17 -in. AppleVision monitor $(1,024 \times 768$ pixel resolution). A chinrest was used to maintain a constant $57-\mathrm{cm}$ viewing distance. Displays were presented and data were collected on PowerMacintosh computers using VScope software (Enns \& Rensink, 1991, Version 1.2.7). Responses were made on a standard keyboard. Displays consisted of 400 rectangular elements (blades of "grass") in a $20 \times 20$ array (see Figure 1A for 3-D arrays and Figure 1B for 2 -D arrays). The array was subdivided into 100 grid locations, each containing four elements. In half of the arrays, one grid location contained a $2 \times 2$ patch of orientation-defined targets.

Each element in the array was jittered randomly from its starting position by $0 \%-30 \%$ of the underlying grid size in the $2-\mathrm{D}$ textures and by half that amount $(0 \%-15 \%)$ in the 3-D textures. Jitter was reduced in the 3-D textures to compensate for the fact that jitter was more noticeable with the upright elements. Each element was a rectangular volume rendered in black $(0 \%$ pixels lit) on a white background $(100 \%$ pixels lit) with an aspect ratio of 0.4 (height $) \times 0.1$ (width) $\times 0.05$ (depth, relevant only in the $3-\mathrm{D}$ arrays). For the $2-\mathrm{D}$ textures, the entire element array subtended $21.5^{\circ} \times 21.5^{\circ}$ of visual angle, and each element subtended $.7^{\circ}$ in height. For the 3 -D textures, the entire display subtended $27.5^{\circ}$ in width at the lower end of the array (depicted as closest to the observer), $10.2^{\circ}$ in width at the upper end of the array (depicted as farthest from the observer), and $9.1^{\circ}$ in height; elements depicted in the closest row of the array subtended $.9^{\circ}$ in height, whereas those depicted in the farthest row subtended $.3^{\circ}$ in height.

For the 2-D textures, background element orientation was tested at 19 different levels, ranging between the two cardinal orientations of $0^{\circ}$ (horizontal) and $90^{\circ}$ (vertical) in $5^{\circ}$ steps. To make testing manageable within a single hour-long session the participants received only half this range, with a cardinality reference of either $0^{\circ}$ or $90^{\circ}$. Thus, one group was tested with background orientations of $0^{\circ}-45^{\circ}$ (10 levels) and the other with background orientations of $45^{\circ}-90^{\circ}$. Within each display, background orientation was uniform. For each level of background orientation, there were 10 target orientation differences ranging from $0^{\circ}$ to $45^{\circ}$ in $5^{\circ}$ steps. The one combination in which target and background orientations were identical (e.g., $10^{\circ}$ target, $10^{\circ}$ background) was the array in which the target was absent. The remaining combinations all yielded a visible target that differed from the background by $5^{\circ}-45^{\circ}$ in orientation. These combinations of background and target orientation resulted in 90 different combinations in which the target was present and 10 different combinations in which the target was absent. In order to encourage a uniform response bias, the total numbers of targetpresent and target-absent trials were equal within a block of trials.

For the 3-D texture gradients, elements were arrayed on a surface that was slanted $65^{\circ}$ away from the horizontal plane of the viewer and rendered using perspective projection (Figure 1A). This surface slant was chosen to yield maximum visibility of 3-D orientation differences. The variation of background and target element orientation was similar to that in the 2-D condition, with the exception that there were now two degrees of freedom for orientation rather than one. As is shown in Figure 1C, 3-D orientation can be described in terms of the parameters $\theta$ and $\phi$. Relative to an upright reference orientation, $\theta$ describes angular deviations from the upright $y$-axis, whereas $\phi$ describes the degree of rotation around the $y$-axis. For convenience, $\phi=0^{\circ}$ indicated an element lying in the frontoparallel plane of the observer, with negative $\phi$ values reflecting a rotation toward the observer and positive $\phi$ values reflecting a rotation away from the observer.

All distractor elements in the 3-D condition were upright $\left(\theta=0^{\circ}\right.$ and $\phi=0^{\circ}$ ) on the depicted surface plane. Target elements were selected to vary over 7 different values of $\theta\left(0^{\circ}, 5^{\circ}, 10^{\circ}, 15^{\circ}, 20^{\circ}, 25^{\circ}\right.$, or $\left.30^{\circ}\right)$ and 11 different values of $\phi\left(45^{\circ}, 35^{\circ}, 25^{\circ}, 15^{\circ}, 5^{\circ}, 0^{\circ},-5^{\circ}\right.$, $-15^{\circ},-25^{\circ},-35^{\circ}$, or $\left.-45^{\circ}\right)$, yielding 77 combinations in total. Of these, 76 were in the target-present condition (either $\theta$ or $\phi$ not $0^{\circ}$ ) and 1 was in the target-absent condition (both $\theta$ and $\phi=0^{\circ}$ ). For each of the 76 target-present combinations, one target was located randomly in each of the surface quadrants, with the restriction that no targets would occur on the boundary edge of the surface (defined as the closest row, the farthest two rows, and each side column of the underlying grid). This yielded 304 target-present displays (76 target-present combinations $\times 4$ quadrant locations).

Procedure. The participants indicated target detection by pressing keys labeled "yes" (the "z" key) and "no" (the "/" key) on a standard keyboard. Half of the participants had the reverse mapping between response and key. The participants were told (1) to keep their eyes at the center of the display on each trial, (2) that half of the displays contained targets, and (3) that they should respond as quickly and as accurately as possible, keeping their errors to $10 \%$ or less. In the 3-D condition, the experimenter used a small rectangular box to demonstrate how targets were defined. To demonstrate the depicted position of the background elements, the box was held upright with the width facing the participant. To demonstrate changes in $\theta$, the box was tilted to the participant's right, away from the $y$-axis at varying angles. To demonstrate changes in $\phi$, the box was kept upright while it was rotated around the $y$-axis. Finally, to illustrate the effects of $\theta$ and $\phi$ varying together, the box was tilted to the right and rotated either away from or toward the participant.

Each trial began with a randomly chosen texture array that remained on the screen until a response had been made or until $7 \mathrm{sec}$ had elapsed. Correct responses were followed by a centrally presented "+" symbol for $600 \mathrm{msec}$, incorrect responses were followed by a "-" symbol, and no response, by a " 0 " symbol. The intertrial interval was $440 \mathrm{msec}$. At the end of each block of trials, a message displayed the percentage of errors for the most recent block of trials, and the participants were prompted to initiate the next block when ready.

The 2-D condition consisted of a total of 540 trials divided into nine blocks of 60 trials. The 90 target-present combinations were evenly distributed among one half of the trials, whereas the 10 targetabsent combinations were evenly distributed among the remaining half. The order of conditions was randomized across trials.

The 3-D condition consisted of 608 trials divided into eight blocks of 76 trials. The 76 target-present combinations were evenly distributed among one half of the trials, whereas the target-absent combination constituted the remaining half. The order of conditions was randomized across trials.

\section{Results}

The primary findings were that (1) target detection performance in 2-D and 3-D textures was influenced comparably by similar orientation differences and (2) detection performance in 3-D textures was sensitive to depicted 3 -D orientation, over and above any differences in 2-D orientation.

Target detection in 2-D and 3-D texture gradients. The mean proportions of detection errors on target-present trials are shown in Figure 2. Errors for 2-D textures are shown in Figure 2A as a function of the angular difference between target and background element orientation. Errors for 3-D textures are shown in Figure 2B as a function of target-background differences in both 3-D $\theta$ and 3-D $\phi$. As is shown in these figures, 2-D tilt has an influence on detection errors comparable to that of 3-D $\theta$, with differences in 3-D $\phi$ playing only a minor role. From a $5^{\circ}$ to a $20^{\circ}$ difference between target and background items, detection errors decreased by $82 \%$ for $2-\mathrm{D}$ displays and by $70 \%$ for 3-D displays. In addition, the mean proportion detection errors on target-absent trials was significantly lower for 2-D textures $(4 \%)$ than for 


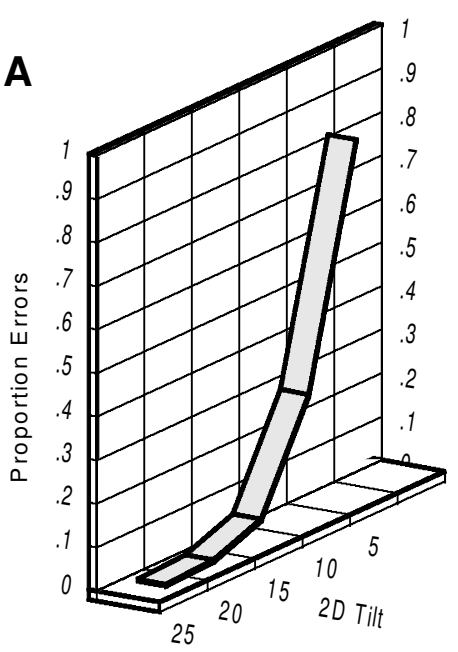

2D Arrays

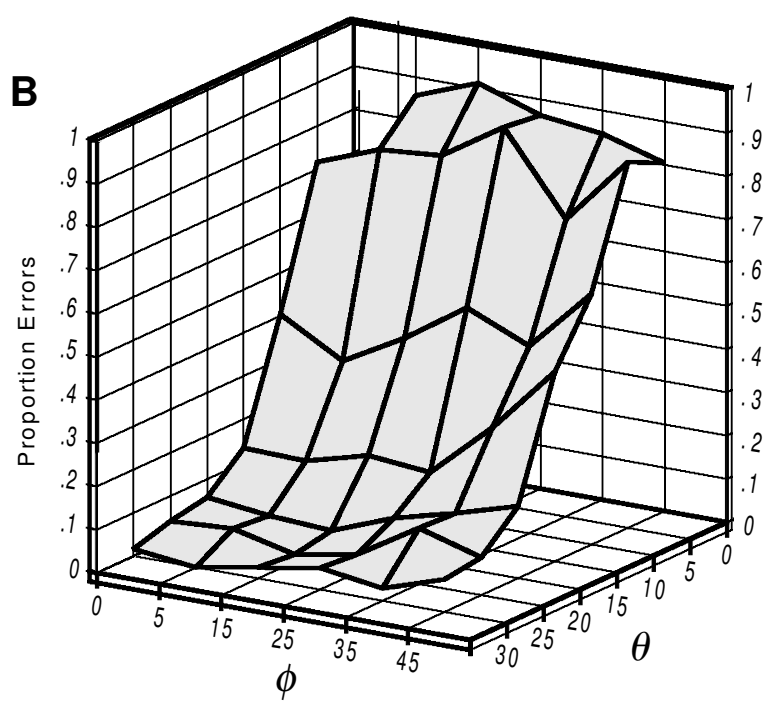

3D Arrays

Figure 2. (A) Detection errors on target-present 2-D textures, plotted as a function of angular difference between target and background orientation (2-D tilt). (B) Detection errors on target-present 3-D textures, plotted as a function of $\theta$ and $\phi$.

3 -D textures $[13 \% ; F(1,48)=47.97, p<.0001]$. Thus, whereas the participants were unlikely to falsely report targets in both conditions, accuracy on target-absent trials was better for 2-D textures.

2-D textures. Detection errors for 2-D textures were analyzed with a mixed-design analysis of variance (ANOVA) involving three factors. Cardinality was a 2-level betweengroups factor in which the reference cardinal orientation in the background was either vertical $\left(0^{\circ}\right)$ or horizontal $\left(90^{\circ}\right)$. Background orientation was a 10-level withinparticipants factor in which the background elements were oriented in increasing steps relative to the cardinal orientations of $0^{\circ}, 5^{\circ}, 10^{\circ}, 15^{\circ}, 20^{\circ}, 25^{\circ}, 30^{\circ}, 35^{\circ}, 40^{\circ}$, or $45^{\circ}$. Target orientation was a 6-level within-participants factor describing how targets differed from the background orientation by $0^{\circ}, 5^{\circ}, 10^{\circ}, 15^{\circ}, 20^{\circ}$, or $25^{\circ}$. Larger differences were not tested, because detection errors were negligible by $25^{\circ}$ of difference.

As is shown in Figure 2A, 2-D detection errors decreased significantly with target orientation $[F(5,90)=$ $429.11, p<.001]$. The improvement with increasing target orientation was very rapid, so that the error rate decreased from a high of $83 \%$ at the smallest target orientation $\left(5^{\circ}\right)$ to less than $6 \%$ errors when target orientation was at $15^{\circ}$.

A main effect of background orientation $[F(9,162)=$ $19.27, p<.001]$ reflected the finding that fewer errors were made when the background elements were in the two cardinal orientations. Simple effects showed that the $0^{\circ}$ background-orientation condition yielded significantly fewer errors $(12.1 \%)$ than all other orientations combined $[21.5 \% ; F(1,162)=77.17, p<.0001]$. This advantage for cardinal backgrounds is often seen in visual search (see, e.g., Treisman \& Gormican, 1988; Wolfe,
Friedman-Hill, Stewart, \& O'Connell, 1992). Vertical and horizontal orientations appear to be privileged by the visual system, so that deviations from cardinality are detected more readily than same-size deviations from noncardinal values.

There was no main effect of cardinality on detection errors $[F(1,18)=.05, p=.83]$. Significant interactions of cardinality with background orientation $[F(9,162)=$ $6.26, p=.0001]$ and with background and target orientation $[F(45,810)=3.10, p=.0001]$ did not reflect any systematic group differences.

3-D textures. Detection errors for 3-D textures were analyzed with a repeated-measures ANOVA involving 3 factors: $\theta$ ( 7 levels: $0^{\circ}, 5^{\circ}, 10^{\circ}, 15^{\circ}, 20^{\circ}, 25^{\circ}$, or $\left.30^{\circ}\right), \phi$ magnitude $\left(6\right.$ levels: $0^{\circ}, 5^{\circ}, 15^{\circ}, 25^{\circ}, 35^{\circ}$, or $\left.45^{\circ}\right)$, and $\phi$ direction (2 levels: negative spin and positive spin).

As can be seen in Figure 2B, detection errors decreased sharply as $\theta$ increased $[F(6,174)=797.67, p=$ .0001 ], from highs of $86 \%$ errors on target-present trials when there were differences only in $\phi\left(\theta=0^{\circ}\right)$ and $78 \%$ errors when the difference in $\theta$ was $5^{\circ}$, to only $9 \%$ errors when the difference in $\theta$ was $20^{\circ}$.

Detection errors tended to increase as $\phi$ magnitude deviated from $0^{\circ}[F(5,145)=84.95, p=.0001]$, averaging $23 \%$ on target-present trials when $\phi$ was $0^{\circ}$ and increasing to $43 \%$ when $\phi$ was most extreme $\left( \pm 45^{\circ}\right)$. This is to be expected, since an increase in $\phi$ corresponds to increased foreshortening of the viewing angle for orientation differences in the display.

The $\theta \times \phi$ magnitude interaction was also significant $[F(30,870)=30.16, p=.0001]$, reflecting the fact that errors were near ceiling and near floor for low $\left(0^{\circ}, 5^{\circ}\right)$ and high $\left(20^{\circ}, 25^{\circ}, 30^{\circ}\right)$ values of $\theta$, respectively. This 
meant that the effects of $\phi$ magnitude were evident only at intermediate values of $\theta$.

$\phi$ direction had a significant influence on detection errors $[F(1,29)=20.34, p=.0001]$, with fewer detection errors made for targets with negative $\phi(33 \%)$ than for those with positive $\phi(35 \%)$.

Sensitivity to 3-D factors. Although the preceding analyses showed that orientation sensitivity was similar for 2-D and 3-D displays, it did not address the question of whether target detection in 3-D displays was really influenced by 3-D factors or merely by 2-D picture-plane differences. The geometry of these textured displays is such that for any given target-background orientation difference, the angular orientation in the picture plane (2-D difference) can be derived from the 3-D parameters of $\theta, \phi$, and viewing angle (Stevens, 1983). Sensitivity to $\theta$ and $\phi$ in target detection could be merely reflecting the combined influence of these factors on the 2-D differences between target and background orientation. On the other hand, if the observers are processing these displays as 3-D scenes, then they should be sensitive to $\theta$ and $\phi$ separately.

The question of which variables the observers were using in the 3-D displays was addressed using a multiple regression analysis. Detection error on target-present trials was the dependent variable. The predictor variables included 2-D tilt (orientation difference between target lines and their local background in the picture plane), 3 -D $\theta, \phi$, and $\phi$ direction. An initial simultaneous regression model revealed that, together, these four predictors accounted for $86 \%$ of the variance in detection errors $[F(4,71)=109.76, p<.001]$. When the individual regression parameters for this model were examined, only two of the factors accounted for a significant portion of variance: $\theta[t(71)=-5.32, p<.001]$ and $\phi[t(71)=$ $2.42, p<.02]$. Importantly, $2-\mathrm{D}$ tilt was not a significant contributor to this model $[t(71)=.32, p=.75]$.

The partial correlations among these factors are shown in Table 1. Although 2-D tilt and 3-D $\theta$ are highly correlated, 2-D tilt plays a negligible role in predicting detection errors when $\theta$ and $\phi$ are held constant. In contrast, $\theta$ and $\phi$ together account for almost as much variance $\left(R^{2}=\right.$ $.859)$ in detection errors as all four predictors taken together $\left(R^{2}=.861\right)$, which indicates an insignificant difference between models $[F(2,71)=0.27, p>.25]$. The reduced regression model, containing only the predictors of $\theta$ and $\phi$, was highly significant $[F(2,73)=222.24, p<.001]$.

\section{Discussion}

In this experiment, it was verified that visual search is sensitive to the depicted 3-D relations in the textured displays. First, the results showed that orientation sensitivity was comparable for orientation differences (tilt) in 2-D displays and for one of the components of object orientation $(\theta)$ in 3-D displays.

Second, and most importantly, even though the variable of 2-D tilt could account for target detection quite well in the 3-D displays, and even though 2-D tilt was strongly correlated with 3-D $\theta$, only $\theta$ accounted for a significant portion of the variance in detection performance when
Table 1

Partial Correlations Between Detection Errors and Various Predictor Variables for the 3-D Displays in Experiment 1

\begin{tabular}{lccccc}
\hline \multicolumn{1}{c}{ Predictor } & Detection & & & & \\
\hline Errors & 2-D Tilt & $\theta$ & $\phi$ & $\phi$ Direction \\
2-D tilt & - & .037 & -.534 & .276 & .114 \\
$\theta$ & & - & .840 & -.526 & -.454 \\
$\phi$ & & & - & .566 & .430 \\
$\phi$ direction & & & & - & -.264 \\
\hline
\end{tabular}

both of these predictors were entered into a regression model. This indicates that search in the present study was based on the depicted 3-D orientation of the elements, and not on the 2-D differences in element orientation. Thus, the conclusions derived from previous studies of visual search using sparse displays, large objects, and large orientation differences (Aks \& Enns, 1996; Enns \& Rensink, 1990a, 1990b) appear to generalize quite well to densely textured arrays of elements depicted on a 3-D surface.

Third, the 3-D $\phi$ component of orientation also had an influence on detection performance, although a smaller one than did the 3-D $\theta$ component. Its influence was in the direction opposite that of $\theta$, with increases in $\phi$ magnitude being associated with an increase in detection errors. This effect likely reflects the increased foreshortening of elements that occurs when $\phi$ magnitude is increased. In the extreme case $\left(\phi=45^{\circ}\right)$, the $\theta$ component of element orientation would be very difficult to discern, because the element would be falling directly away from or toward the viewer. In everyday viewing, the height of tall buildings and mountains is routinely underestimated for exactly this reason.

\section{EXPERIMENT 2 A Direct-Action Advantage}

The purpose of this experiment was to examine detection accuracy along with two different modes of target localization: direct pointing to the target location on the screen and indirect pointing to a keypad that spatially corresponded to the screen locations. In traditional feature integration theory, location information is thought to follow detection, but the theory does not provide a way to address whether localization action has any effects on performance (Treisman \& Gelade, 1980).

A hypothesis derived from the dual systems theory of Goodale and Milner (1992; Milner \& Goodale, 1995) is that target localization accuracy would be greater for direct responses than for indirect ones, since the former could be guided by the action-oriented dorsal pathway. Indirect responses, in contrast, would have to be guided by the less accurate but more consciously accessible ventral pathway.

Previous support for the greater spatial sensitivity of the dorsal pathway has come from studies in which perceptual judgments of various visual illusions were compared with responses involving direct action (Brenner \& 
Smeets, 1996; Jackson \& Shaw, 2000). For example, although observers report seeing the Ebbinghaus illusion (a target disk surrounded by smaller disks appears larger than the same target disk surrounded by larger disks), their reach and grasp of a 3-D target disk is scaled to the objective, and not to the perceived, size of the disk (Aglioti, DeSouza, \& Goodale, 1995). It has also been demonstrated that manual aiming movements are sensitive to target displacements under some conditions, even though observers are unaware of these displacements (Fecteau, Chua, Franks, \& Enns, 2001; Goodale, Pélisson, \& Prablanc, 1986). In the present experiment, we tested whether a similar dissociation between awareness and action would be found between direct and indirect modes of localization during visual search.

One main change was made to the procedures used in Experiment 1 . In the present experiment, immediately following the detection response (target presence vs. target absence), the search display was replaced by the response grid shown in Figure 3A. This grid remained in view until a localization response was made. In addition to examining localization performance as a function of differences in target and background orientation, performance was examined at three levels of spatial resolution (cell, quadrant, and hemifield) to determine whether response mode differences interacted with spatial resolution.

\section{Method}

Participants. Fifty-two undergraduate students (41 females, 11 males; mean age $=19.6$ years) participated in exchange for course credit. All reported normal or corrected-to-normal vision. Twenty-six students participated in the direct-localization condition (14 in the positive $\phi$ condition and 12 in the negative $\phi$ condition; see the Stimuli and Design section for details). The other 26 students participated in the indirect-localization conditions (13 in the positive $\phi$ condition and 13 in the negative $\phi$ condition).

Stimuli and Design. The search displays and timing for the detection task were the same as in the 3-D condition of Experiment 1, with the exception that the viewing distance from the monitor was $50 \mathrm{~cm}$. Following the detection response on target-present displays, the search display was replaced by a black and white outline grid of the surface plane on which the elements were located (Figure $3 \mathrm{~A}$ ). This display was rendered using the same perspective projection as in Experiment 1. The plane was divided into a $4 \times 4$ grid that subdivided 64 possible target locations into 16 regions. Each region was labeled with a letter ranging from A to $\mathrm{P}$, starting from the top left region and following left to right to the bottom right region. This grid was surrounded by an empty frame that corresponded to the boundary edge, where no targets were presented. Target-absent displays were followed by a centered line of the text: "Press the space bar to continue.'

To limit the testing session to $1 \mathrm{~h}$, the $7 \theta \times 11 \phi$ values used in Experiment 1 were split into two between-groups conditions according to $\phi$. In the positive $\phi$ group, target elements varied over seven values of $\theta\left(0^{\circ}, 5^{\circ}, 10^{\circ}, 15^{\circ}, 20^{\circ}, 25^{\circ}\right.$, and $\left.30^{\circ}\right)$ and 6 values of $\phi\left(0^{\circ}, 5^{\circ}, 15^{\circ}, 25^{\circ}, 35^{\circ}\right.$, and $\left.45^{\circ}\right)$, yielding 42 combinations in total. Of these, 41 constituted the target-present condition (either $\theta$ or $\phi$ not $0^{\circ}$ ) and 1 constituted the target-absent condition (both $\theta$ and $\phi=$ $\left.0^{\circ}\right)$. For each of the 41 combinations in the target-present condition, one target patch was located randomly in each of the surface quadrants, with the restriction that no targets would occur on the boundary edge of the surface (defined as the closest row, the farthest row, and each side column of the underlying grid). This yielded 164 target-present trials $(41$ target-present combinations $\times 4$ quadrant

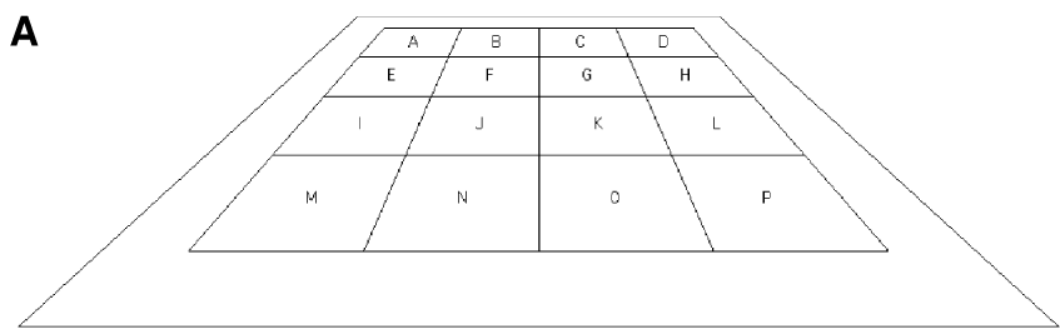

B

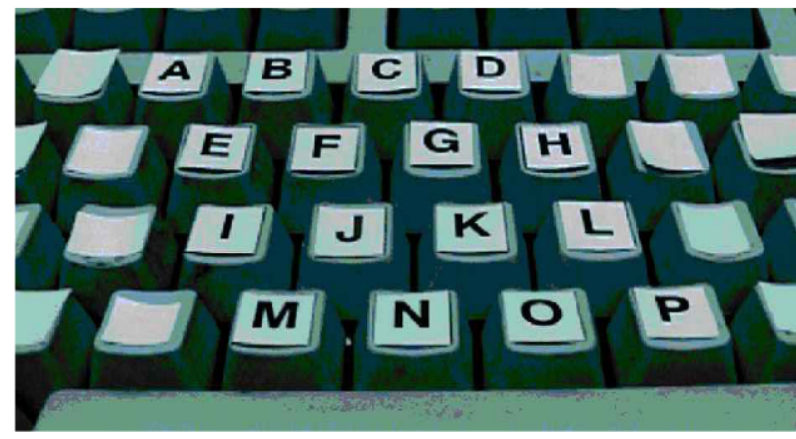

Figure 3. (A) The surface corresponds exactly in size to the surface of the search array in Figure 1A. It has 16 possible target locations, marked with the letters A-P. (B) Response keys are spatially mapped to the configuration of possible target locations. 
locations). The same specifications were used in the negative $\phi$ condition, in which the seven values of $\theta$ were combined with six values of $\phi\left(0^{\circ},-5^{\circ},-15^{\circ},-25^{\circ},-35^{\circ}\right.$, and $\left.-45^{\circ}\right)$.

Procedure. Task instructions were the same as in Experiment 1 for target detection, and additional instructions were provided for the localization task. These included the information that targetpresent displays were always followed by the location array, which remained on screen until the participant responded. Importantly, the presentation of the location array was contingent on the presentation of a target-present display, and not on the participant's response. Thus, the participants were instructed to use the presence of the localization array as feedback on their detection responses, with the location array indicating a target-present display and the space bar prompt indicating a target-absent display.

The participants indicated a target location either directly or indirectly, depending on their group assignment. If they had responded "no" on a target-present trial (a miss in the detection task), they were instructed to make their best guess of target location.

In the direct response group, the participants used their dominant hands to point to the grid location containing the target in the preceding 3-D array. Following this pointing response, they used the keyboard to record the letter that corresponded to the grid square to which they had just pointed. The keyboard was labeled with the 16 letters from the location array (Figure 3B), spatially corresponding as closely as possible to the locations of the letters on the screen. The remaining keys were covered with blank labels to reduce the possibility of responding with nondesignated keys.

In the indirect response group, the procedure was the same, except that the pointing response was omitted. When the location grid appeared, localization responses were made by simply pressing the labeled key that corresponded to the depicted target location. Thus, this condition required less overall action than the direct-action condition did and, importantly, required a response that was only indirectly mapped to the target location. It is important to note that observers in both localization conditions needed to consult the labeled squares on the location grid in order to know which labeled key to press. The requirement of a keypress in both conditions was included to control for spatial layout differences between the onscreen target locations and the response keys.

Each of the four between-groups conditions (direct localizationpositive $\phi$, direct localization-negative $\phi$, indirect localizationpositive $\phi$, indirect localization-negative $\phi$ ) consisted of 328 trials divided into eight blocks of 41 trials. The 41 target-present combinations were evenly distributed among one half of the trials, whereas the target-absent combinations constituted the remaining half. The order of conditions was randomized across trials.

\section{Results}

The main finding of this experiment was that localization performance was better for direct- than for indirectaction responses at all levels of spatial resolution.

Target detection. A preliminary analysis was conducted on detection errors to confirm that the observers were sensitive to depicted 3-D orientation, as they had been in Experiment 1. Detection errors were analyzed with a mixed-design ANOVA that included the two within-participants factors of $\theta\left(0^{\circ}, 5^{\circ}, 10^{\circ}, 15^{\circ}, 20^{\circ}, 25^{\circ}\right.$, or $\left.30^{\circ}\right)$ and $\phi$ magnitude $\left(0^{\circ}, 5^{\circ}, 15^{\circ}, 25^{\circ}, 35^{\circ}\right.$, or $\left.45^{\circ}\right)$ and the two between-groups factors of $\phi$ direction (positive or negative) and action (direct or indirect).

The influence of $\theta$ and $\phi$ magnitude on detection errors closely resembled that of 3-D textures in Experiment 1 [average target-present errors $=37 \%$, target-absent errors $=15 \%$; significant effects of $\theta, F(6,294)=781.54$, $p=.0001$; significant effects of $\phi$ magnitude, $F(5,245)=$ $107.24, p=.0001$; significant effects of $\theta \times \phi$ magnitude, $F(30,1470)=34.09, p=.0001]$. As $\theta$ increased, detection errors decreased. As $\phi$ magnitude increased, errors increased, but only for intermediate values of $\theta$. This was a replication of the patterns of detection performance seen in Experiment 1.

The between-groups factor of $\phi$ direction was again significant $[F(1,49)=4.63, p=.04]$, with fewer detection errors made for targets with negative $\phi(35 \%)$ than for those with positive $\phi(39 \%)$.

There was a significant $\theta \times$ action interaction $[F(6,294)=2.22, p=.04]$. For $\theta=15^{\circ}, 20^{\circ}$, and $25^{\circ}$, the direct-action group had fewer detection errors than the indirect-action group. There were no other significant effects of action.

Influence of 2-D versus 3-D factors. To confirm the finding of Experiment 1 that 3-D $\theta$ and $\phi$ were the main factors necessary to account for target detection, detection errors in the present experiment were also examined as a function of 2-D tilt, 3-D $\theta, \phi$, and $\phi$ direction in a simultaneous regression model. The four predictors together accounted for $86 \%$ of detection variance $[F(4,77)=$ $119.99, p<.0001]$; however, only two factors accounted for significant portions of variance: $\theta[t(77)=-4.02, p=$ $.0001]$ and $\phi[t(77)=2.26, p=.03]$. These two predictors alone accounted for almost as much variance $\left[R^{2}=\right.$ $.858, F(2,79)=238.87, p<.0001]$ as all four predictors together $\left(R^{2}=.862\right)$, an insignificant difference between models $[F(2,77)=.06, p>.25]$. These results therefore replicate the finding of Experiment 1 that detection performance was based primarily on the depicted 3-D orientation of the elements, and not on 2-D orientation.

Sensitivity and criterion levels. A signal detection analysis was used to examine detection discriminability separately from any detection biases that observers may have had. This seemed particularly important because the prior analysis revealed some differences in detection sensitivity between the two action groups, and subsequent analyses showed that these groups differed in localization accuracy as well. Detection sensitivity $\left(d^{\prime}\right)$ and response criterion $(\log \beta)$ were therefore calculated for each participant. These measures were analyzed with an ANOVA involving the between-groups factor of action (direct or indirect).

The analysis of detection sensitivity revealed that the observers were significantly more sensitive in the directaction group $\left(d^{\prime}=1.57\right)$ than in the indirect-action group $\left[d^{\prime}=1.29, F(1,50)=10.24, p=.002\right]$. This greater sensitivity is consistent with the fewer errors made by the directaction group in the previous analysis. However, one possibility is that this greater sensitivity came about because response demands of the direct-localization task elicited a more liberal response bias. This possibility was examined with an analysis of $\beta$ criterion levels, which revealed that the observers in the direct-action group were also significantly more conservative (i.e., they used a more stringent criterion for detection) than those in the 
indirect-action group $[\beta=2.25, \log \beta=.30$ and $\beta=1.68$, $\log \beta=.20$, respectively; $F(1,50)=4.28, p=.04$, ANOVA performed on $\log \beta$ ]. This confirmed that the greater sensitivity of the observers in the direct-action group was not also associated with a more liberal response bias. In fact, target detection in the direct-action group was both more sensitive and more conservative than that in the indirect-action group. This finding may reflect inherent group differences (despite random assignment of participants) or the differential influence of the action requirements of the task.

Target localization. Target localization responses were coded using three levels of resolution: cell (only the correct cell in the $4 \times 4$ location matrix was counted as correct), quadrant (any one of the four cells in the correct quadrant was counted as correct), and hemifield errors (any one of the eight cells in the correct hemifield was counted as correct). For each resolution of response, chance levels of error were calculated as 1 minus chance probability of correct response. Chance error rates were therefore $93.8 \%$ for the cell level of resolution, $75 \%$ for the quadrant level, and $50 \%$ for the hemifield level. The target localization errors are shown in Figure 4 for the cell resolution as a function of $\theta$ and $\phi$. Errors are shown separately for direct and indirect responses.

Location errors were analyzed with a mixed-design ANOVA that included the two within-participants factors of $\theta\left(0^{\circ}, 5^{\circ}, 10^{\circ}, 15^{\circ}, 20^{\circ}, 25^{\circ}\right.$, or $\left.30^{\circ}\right)$ and $\phi$ magnitude $\left(0^{\circ}, 5^{\circ}, 15^{\circ}, 25^{\circ}, 35^{\circ}\right.$, or $\left.45^{\circ}\right)$ and the two betweengroups factors of $\phi$ direction (positive or negative) and action (direct or indirect). This analysis was conducted for each of the cell, quadrant, and hemifield resolutions. Because there were no data points for the target-absent trials (i.e., the combination of $\theta=0^{\circ}$ and $\phi=0^{\circ}$ ) and the design was incomplete without these cells, the chance rate of localization errors was used in these cells of the design.

There was a consistent advantage for the direct response at all resolutions of localization, reflected in significant action effects [cell, $F(1,49)=17.69, p=.0001 ;$ quadrant, $F(1,49)=13.49, p=.0006$; hemifield, $F(1,49)=11.98$, $p=.001]$. Fewer errors were made for direct than for indirect action at the cell (57\% vs. $66 \%$ ), quadrant (33\% vs. $39 \%$ ), and hemifield (19\% vs. $24 \%$ ) resolutions.

Localization errors generally resembled detection errors with respect to the influence of $\theta$ and $\phi$ magnitude. Errors decreased with increases in $\theta[F(6,294)=301.74$ (cell), 556.18 (quadrant), and 338.56 (hemifield)] and increased with increases in $\phi$ magnitude $[F(5,245)=$ 52.63 (cell), 50.37 (quadrant), and 8.18 (hemifield)]. The influence of $\phi$ magnitude was most apparent at intermediate values of $\theta$ for all reported effects. Floor and ceiling effects attenuated the effects of $\phi$ magnitude at more extreme values of $\theta$ [significant $\theta \times \phi$ magnitude interaction: $F(30,1470)=29.96$ (cell), 13.68 (quadrant), 6.52 (hemifield]); $p=.0001]$.

There was no main effect of $\phi$ direction at any resolution $[F(1,49)=.07$ (cell), 1.71 (quadrant), 1.05 (hemifield), $p>.20$ ], although there was a significant interaction between $\theta \times \phi$ direction at all three resolutions [cell, $F(6,294)=2.39, p=.03 ;$ quadrant, $F(6,294)=$ $4.43, p=.0003$; hemifield, $F(6,294)=4.36, p=.0003]$. As with detection errors, these effects appeared to reflect the generally greater sensitivity to negative directions of $\phi$, which interacted with floor and ceiling effects to produce the statistically significant interactions.

The $\theta \times$ action interaction was also significant at all resolutions of localization [cell, $F(6,294)=3.50, p=.002$; quadrant, $F(6,294)=3.24, p=.004$; hemifield, $F(6,294)=$ $3.85, p=.001]$, as was the $\theta \times \phi$ magnitude $\times$ action interaction for cell localization errors $[F(30,1470)=.05$, $p=.006]$. These interactions reflected an attenuation of the effect of action as the result of ceiling effects in the most difficult discrimination conditions (i.e., small values of $\theta$ and large values of $\phi$ ).

Controlling for baseline differences in detection sensitivity and criterion. Because the analysis of detection sensitivity revealed baseline differences in target de-
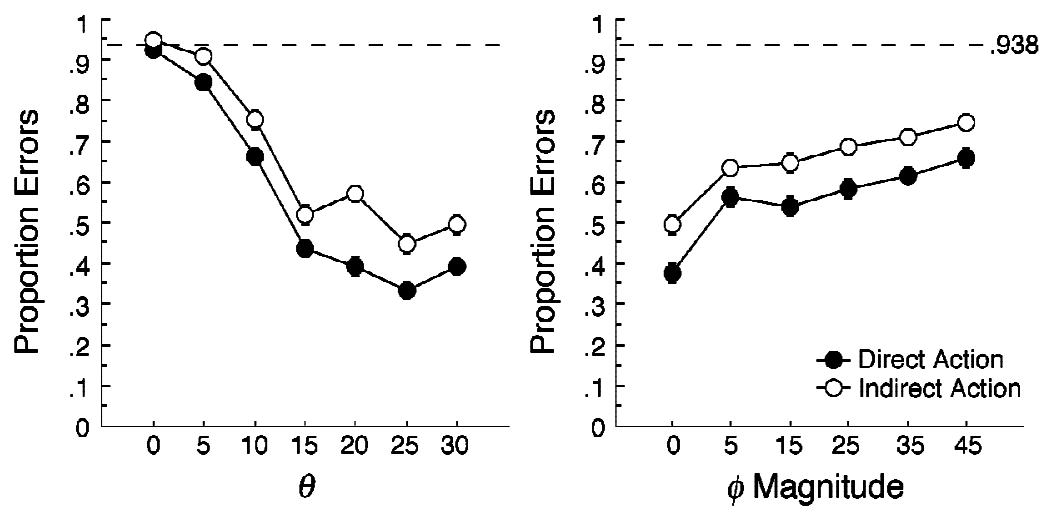

Figure 4. Target localization errors as a function of $\theta$ and $\phi$ magnitude. Errors are shown for the cell resolution of localization. The chance level of localization is indicated by the dashed line. Error bars represent \pm 1 standard error and are smaller than the symbols used to indicate the mean. 
tectability between the two action groups, it raised the possibility that these differences reflected inherent group differences rather than differences resulting from the action requirements of the task. To address this question, participants from both the direct- and indirect-action groups were sampled in order to equate these groups in detection sensitivity prior to an analysis of localization performance. Beginning with 26 participants in each group, the participants with the highest $d^{\prime}$ in the directaction group and the lowest $d^{\prime}$ in the indirect-action group were excluded until $d^{\prime}$ and $\beta$ levels were equated. After 7 participants had been eliminated from each group, the mean $d^{\prime}$ was 1.44 for each group; however, $\beta$ was still higher for the indirect-action $(\beta=1.82)$ than for the direct-action $(\beta=1.73)$ group. To equate for $\beta$ as well, an examination of the $d^{\prime}$ and $\beta$ levels of each participant revealed that excluding the participant with the ninth highest $d^{\prime}$ instead of the one with the seventh highest resulted in a data set with 19 participants per group, with $\beta=1.81$ for the direct-action group, $\beta=1.82$ for the indirect-action group, and $d^{\prime}=1.44$ for both groups.

Localization errors for the remaining participants were examined with a mixed-design ANOVA involving the two within-participants factors of $\theta\left(0^{\circ}, 5^{\circ}, 10^{\circ}, 15^{\circ}\right.$, $20^{\circ}, 25^{\circ}$, or $\left.30^{\circ}\right)$ and $\phi$ magnitude $\left(0^{\circ}, 5^{\circ}, 15^{\circ}, 25^{\circ}, 35^{\circ}\right.$, or $45^{\circ}$ ) and the two between-groups factors of $\phi$ direction (positive or negative) and action (direct or indirect). The general pattern of results remained largely unchanged. Most importantly, the main effect of action was significant at the cell $[F(1,35)=6.59, p=.02]$ and quad$\operatorname{rant}[F(1,35)=4.44, p=.04]$ levels and approached significance at the hemifield level $[F(1,35)=3.22, p=.08]$.

\section{Discussion}

The primary finding of this experiment was a consistent advantage for direct- over indirect-localization responses. The observers were more accurate in localizing targets when the response involved pointing to the screen than when they made only a spatially mapped keyboard response. This occurred at all levels of spatial resolution, indicating that it was not caused by response-mapping difficulty at the finest level of resolution. The advantage is consistent with dual systems theory, in that visually guided actions that originate in the dorsal stream should reveal more precise spatial information than actions originating in the ventral stream (Milner \& Goodale, 1995).

A potential limitation of the direct-action advantage stems from the fact that these results were obtained under conditions in which the observers were always asked to answer two questions about a display: "Was there a target?" and "Where was it?" This being the case, it is unknown whether the direct-action advantage will occur when observers are given only a single question to answer regarding the location of the target. It is possible that the direct-action advantage will be particularly strong when competing cognitive operations must also be coordinated, as is required with the target detection question. This possibility was explored in Experiment 3 .

\section{EXPERIMENT 3 \\ Task Sharing Between Target Detection and Target Localization}

The results of the previous experiment raised the possibility that different cognitive resources are used to perform direct and indirect localization. J. Norman (2002) reasons that if the dorsal and ventral streams can function independently, with the dorsal stream requiring little or no conscious control, then the two streams may be able to carry out two tasks simultaneously with little interference from one another. However, this prediction runs contrary to many dual-task studies that have consistently shown that there is a cost when the observer is asked two questions about a single visual display. For example, when observers try to detect targets that differ in their orientation by $90^{\circ}$ from background elements, they are unable to do this concurrently with the task of detecting a target letter in a rapid serial stream (Di Lollo et al., 2001; Joseph, Chun, \& Nakayama, 1997).

The dual-task cost is also usually reflected in reduced performance on the task that is performed second (Braun \& Sagi, 1990; Di Lollo et al., 2001). This is attributed to attentional resources' being engaged by the first task and, thus, unavailable for the second task. In accordance with this idea, if the second task is designed to demand less attention, then the presence of the first task has a smaller effect on performance on the second task. Sometimes the two tasks can be designed to be so easy that they can be performed in concert without any apparent costs at all. For example, neither the detection nor the coarse localization of an orientation-defined target suffers when combined with the primary task of also detecting or coarsely localizing a similar target (Braun \& Sagi, 1990, 1991). This makes it appear as though the limiting factor on dual-task performance is the extent to which each of the tasks draws on a common, limited pool of cognitive resources.

To address the extent to which the difference in directand indirect-localization performance depended on whether dorsal or ventral resources were available, two new comparisons were made in Experiment 3. First, detection, direct localization, and indirect localization were tested as single tasks so that performance could be compared with that on the dual tasks of Experiment 2 (i.e., detection followed by direct localization and detection followed by indirect localization). If task sharing is enhanced when two tasks rely on different streams, then performance on the dual task of detection and direct localization (tapping ventral and dorsal streams, respectively) should suffer the least, relative to performance on the single-task control conditions.

Second, the order of the two tasks in Experiment 2 was reversed so that direct or indirect localization was performed first, followed by the detection task (indirect localization followed by detection, direct localization followed by detection). This permitted an examination of task order effects. Since dual-task costs are typically 
manifested in the second task, we reasoned that indirect localization accuracy would suffer less if it were the first of the two tasks performed rather than the second, as it was in Experiment 2.

\section{Method}

Participants. Fifty-three undergraduate students (38 females, 15 males; mean age $=21.9$ years) participated in exchange for course credit. All reported normal or corrected-to-normal vision. For the single-task controls, 11 students participated in the detectionalone condition, 10 in the direct-localization-alone condition, and 10 in the indirect-localization-alone condition. For the dual-task controls, 11 students participated in the direct-localization-f irst condition and 11 in the indirect-localization-f irst condition.

Stimuli, Design, and Procedure. For the single-task controls, the method was the same as in Experiment 2, with the exception that the participants were given only one task. In the detection-alone condition, the response to the search display was followed by a "+" symbol at the center of the screen on correct responses, a "-" symbol on incorrect responses, and a "0" symbol if 7 sec elapsed without a response. Only half of the displays in the detection-alone condition contained a target. In the two localization-alone conditions, the search display was presented for $7 \mathrm{sec}$, during which time the participant was not permitted to respond. This was followed by the localization response grid, which was the signal for the participant to respond. Every display in the localization conditions contained a target.

The detection-alone condition consisted of 608 trials divided into eight blocks of 76 trials. The 76 target-present combinations were evenly distributed among one half of the trials, whereas the targetabsent combinations were evenly distributed among the remaining half. The order of conditions was randomized across trials. The two localization-alone conditions each consisted of 304 trials divided into eight blocks of 38 trials.

For the reversed dual task, the method was also the same as in Experiment 2, with the primary exception that the localization task was performed first on each trial. In addition, only negative $\phi$ search displays were used, in order to limit the testing session to approximately $1 \mathrm{~h}$. Search displays were presented for $7 \mathrm{sec}$ and followed by the localization response grid, which signaled the participants to make a localization response. The participants were instructed to make the appropriate localization response for every search display regardless of target presence or absence. After making the direct- or indirect-localization response, the grid display was replaced by a question mark, which prompted the detection response. The participants were instructed to indicate whether they believed a target had actually been present in the search array by making the "yes" or "no" detection response. It was emphasized that only $50 \%$ of the search arrays contained targets.

As in Experiment 2, the reversed dual-task conditions each consisted of 328 trials divided into eight blocks of 41 trials. The 41 target-present combinations were evenly distributed among one half of the trials, whereas the target-absent combination was evenly distributed among the remaining half. The order of conditions was randomized across trials.

\section{Results}

The main finding was that the difference between direct and indirect target localization was largest when localization was performed following target detection. Although there was a trend toward better direct localization in the single-task control conditions and in the reverse dual-task conditions, it was not statistically significant. Furthermore, a comparison of all three conditions revealed that the difference in accuracy is best seen as an impairment in the performance of the indirect-localization task after a target detection response has been made.

Target detection for single and dual tasks. The target detection errors for single and dual tasks are shown in Figure 5. Detection errors in the single-task condition averaged 38\% for target-present trials and $15 \%$ for targetabsent trials, which is very similar to the error rate for the dual-task detection-first condition in Experiment 2. Detection errors in the reverse dual-task condition (detection second) averaged $28 \%$ for target-present trials and $20 \%$ for target-absent trials, which is a lower rate of errors than for the other conditions. Detection errors for all single- and dual-task conditions were analyzed with a mixed-design ANOVA involving four factors: the two between-groups factors of action (direct localization, indirect localization, or none) and task order (detection first, second, or alone) and the two within-participants factors of $\theta\left(0^{\circ}, 5^{\circ}, 10^{\circ}, 15^{\circ}, 20^{\circ}, 25^{\circ}\right.$, or $\left.30^{\circ}\right)$ and $\phi$ magnitude $\left(0^{\circ}, 5^{\circ}, 15^{\circ}, 25^{\circ}, 35^{\circ}\right.$, or $\left.45^{\circ}\right)$.

The analysis revealed no main effect of action $[F(1,80)=$ $2.38, p=.13$ ], nor did action interact significantly with other factors. Although there was a slight advantage for detection paired with direct action in Experiment 2, this difference was no longer significant when the analysis included both single- and dual-task detection. There was a main effect of task order $[F(1,80)=34.49, p=.001]$, reflecting generally lower errors when target detection was the second task. Task order also interacted significantly with $\theta[F(6,480)=15.28, p=.0001], \phi$ magnitude $[F(5,400)=5.69, p=.0001]$, and $\theta \times \phi$ magnitude $[F(30,2400)=2.11, p=.001]$. All of these effects reflected generally fewer errors, and hence smaller effects

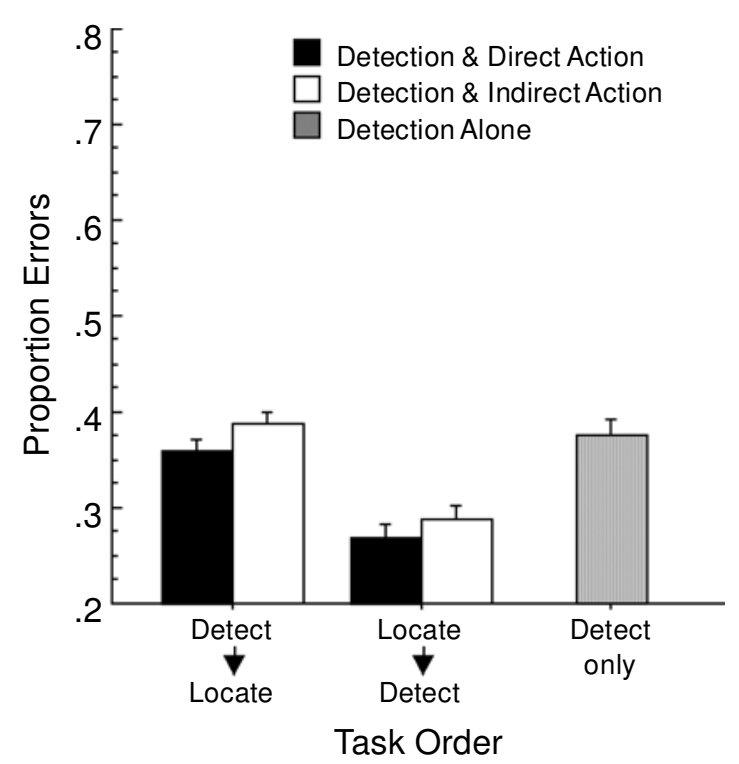

Figure 5. Detection errors for dual-task (detection followed by localization, localization followed by detection) and single-task (detection-alone) conditions, collapsed across $\theta$ and $\phi$ magnitudes. The chance level of detection is $50 \%$. Error bars represent \pm 1 standard error. 
of intervening factors, when target detection was performed after target localization.

There is a possibility that detection sensitivity levels may have been affected by action in a way that was not evident in the analysis of mean errors. There is also a possibility that the improvement in detection when it was the task performed second was due to criterion shifts rather than to sensitivity. To examine these questions, a signal detection analysis was performed for single-task, second-task (Experiment 3), and first-task (Experiment 2) detection. Sensitivity and criterion levels were analyzed with an ANOVA involving the two between-groups factors of action and task order.

There was a main effect of action on sensitivity $[F(1,80)=4.55, p=.04]$, reflecting higher mean sensitivity for the direct-action group $\left(d^{\prime}=1.58\right)$ than for the indirect-action $\left(d^{\prime}=1.34\right)$ and detection-alone $\left(d^{\prime}=1.42\right)$ groups $[F(1,80)=4.45, p=.04]$. There was no significant difference in mean sensitivity between the indirectaction groups and the detection-alone group $[F(1,80)=$ $.11, p=.74]$. In addition, there was no effect of task order on sensitivity. The analysis of criterion levels revealed no effects of task order or action $(p>.1)$.

Target localization for single and dual tasks. The mean target localization errors are shown in Figure 6. Localization errors were analyzed with a mixed-design ANOVA containing the two between-groups factors of task order (localization first, second, or alone) and action (direct or indirect) and the two within-participants factors of $\theta\left(0^{\circ}, 5^{\circ}, 10^{\circ}, 15^{\circ}, 20^{\circ}, 25^{\circ}\right.$, or $\left.30^{\circ}\right)$ and $\phi$ magnitude $\left(0^{\circ}, 5^{\circ}, 15^{\circ}, 25^{\circ}, 35^{\circ}\right.$, or $\left.45^{\circ}\right)$. The analysis revealed that, relative to single- and first-task controls, indirectlocalization accuracy suffered as a second task, but direct localization accuracy did not. There was a significant effect of task order at all levels of location resolution [cell, $F(2,88)=6.12, p=.003$; quadrant, $F(2,88)=5.91, p=$ .004 ; hemifield, $F(2,88)=6.91, p=.002]$. Means comparisons revealed that localization as a second task was consistently more error prone than localization as a first or single task [cell, $F(1,88)=12.07, p=.0008$; quadrant, $F(1,88)=11.78, p=.0009$; hemifield, $F(1,88)=11.36$, $p=.001]$. Finer grain comparisons showed further that this trend was entirely driven by greater errors for indirect than for direct localization. Errors for the indirectlocalization-second condition were significantly greater than those for the other conditions [cell, $F(1,88)=25.49$, $p=.001$; quadrant, $F(1,88)=24.65, p=.001$; hemifield, $F(1,88)=24.82, p=.001]$, whereas errors for the directlocalization-second condition were not significantly different at any level of resolution $(p>.45)$.

In Experiment 2, a significant advantage was established for direct action when localization was performed after detection. In the present comparison involving all task and order conditions, this action advantage was still significant, but it was attenuated by the inclusion of single and reversed dual-task conditions [cell, $F(1,88)=$ $10.84, p=.001$; quadrant, $F(1,88)=8.14, p=.005]$. When the same comparison was made for only the con-

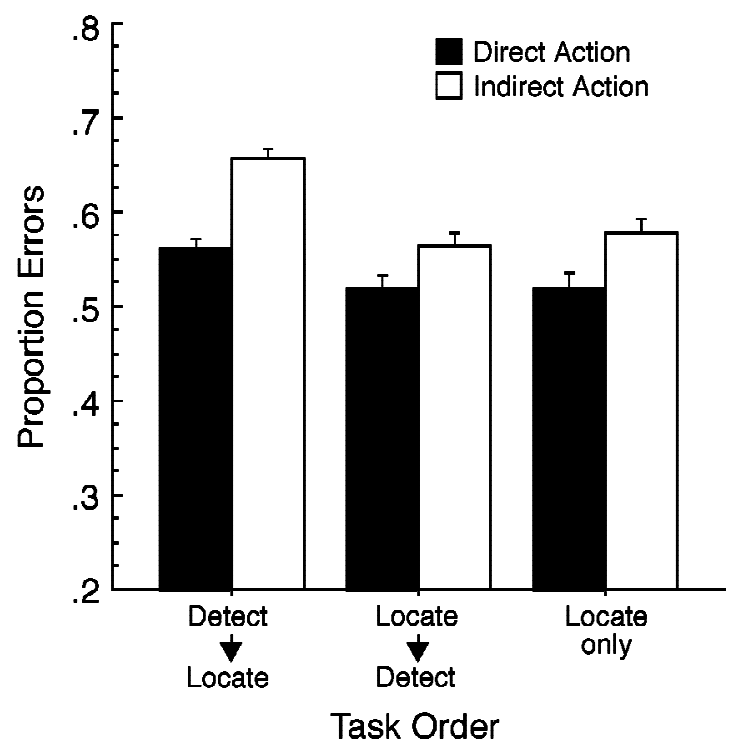

Figure 6. Target localization errors for dual-task (detection followed by localization, localization followed by detection) and single-task (localization alone) conditions, collapsed across $\theta$ and $\phi$ magnitudes. Errors are shown for the cell resolution of localization. The chance level of localization is $93.8 \%$. Error bars represent \pm 1 standard error.

ditions in which localization was performed alone or as the first task, the action effect was not significant at any level of spatial resolution $(p>.05)$, nor did it interact with task order. There was, however, a significant $\theta \times$ action interaction [cell, $F(6,228)=2.25, p=.04$; quadrant, $F(6,228)=2.17, p=.05$ ], which reflected a small but significant direct-action advantage at intermediate values of $\theta$. At levels of $\theta$ that were either very easy (e.g., $25^{\circ}$ and $30^{\circ}$ ) or very difficult (e.g., $0^{\circ}$ and $5^{\circ}$ ) to discriminate, the action effect was not significant.

\section{Discussion}

The question explored in Experiment 3 was whether interference between target detection and target localization was influenced by the degree to which they both depended on ventral stream processing. The results indicate that there was a cost in localization accuracy when it was performed after target detection, but only when it involved an indirect action. Direct target localization was unaffected by whether or not a detection report was made first, which implies that it does not compete for access to the same attention-limited resources. This is consistent with dual systems theory, which predicts that detection and indirect action both rely on the ventral stream, but that the direct action can be performed largely by the dorsal stream.

A second finding was that differences between direct and indirect action were attenuated when localization was performed as a single task or as the first of two tasks. This suggests that indirect target localization can be performed best when ventral stream resources can be entirely devoted to it. The selective impairment for indirect 
localization following target detection is consistent with previous research showing that it is the second of two tasks that suffers most from resource competition. Yet, it is notable that there was a small, consistent advantage for direct action even under these conditions. This is consistent with the dorsal stream's having somewhat finer spatial sensitivity, even when there is no competition for cognitive resources.

A third finding was that detection sensitivity was higher when paired with direct action than when paired with indirect action or when performed alone. At the same time, there were no sensitivity differences between detection paired with indirect action and detection performed alone. Together, these findings suggest that the need to perform a direct action benefits not only localization but also detection sensitivity. This implies that the ventral stream task of detection has some ability to access the more accurate target information in the dorsal stream.

A final finding was that target detection benefited when it followed both types of target localization (Figure 5), whereas indirect target localization was impaired when it followed target detection (Figure 6). The improvement of detection as the second task is contrary to the general trend in dual-task conditions: impairment of the second task. Furthermore, this finding cannot be attributed to group differences, since the same group of observers was not more accurate in localization than the single-task localization control groups. Instead, this finding suggests a task asymmetry: Whereas target detection can benefit from prior attempts to locate a target, target localization does not benefit from prior efforts to detect a target. Future research will be needed to determine whether this is because target detection by the ventral stream has partial access to the results of dorsal stream processing involved in all kinds of target localization, or whether simply preparing for target localization focuses attention in such a way as to benefit target detection as a secondary effect.

\section{GENERAL DISCUSSION}

In this study, we examined target detection and localization in a visual search task from the perspective of dual visual systems (Goodale \& Milner, 1992; Milner \& Goodale, 1995). The two main questions were as follows: (1) Is target localization influenced by whether visually guided actions are made directly (dorsal stream) or indirectly (ventral stream)? (2) Are target detection and localization easier to perform concurrently when the localization task involves a direct action (dorsal stream)?

Experiment 1 involved a necessary preliminary step in which we determined that visual search for orientationdefined targets was sensitive to depicted 3-D, and not merely to 2-D, orientation differences. This meant that the observers were guiding their visual search using a 3 -D interpretation of the displays.

In Experiment 2, the observers were given the dual tasks of target detection and target localization for the same displays. The question of interest was whether lo- calization accuracy would differ for direct (dorsal stream) versus indirect (ventral stream) actions. The main finding was that direct action (i.e., pointing to the target on the screen) resulted in fewer errors than did indirect action (i.e., pointing to the spatially corresponding location on the keyboard) over a wide range of scoring for spatial resolution. This is consistent with dual visual systems theory, which predicts that visually guided actions made directly on an object can be more accurate than actions made indirectly to the same visual input.

In Experiment 3, target localization was tested under conditions in which all cognitive resources could be devoted to localization, either because it was the only task to be performed or because it was the first of two tasks. The main finding was that only indirect target localization showed significant costs in the dual-task condition, and it did so only when it followed target detection. This is consistent with the prediction of dual systems theory that target detection, which is thought to depend on the ventral stream, shares few cognitive resources with visually guided direct actions, since these can be performed using the dorsal stream.

\section{What's Behind the Direct-Action Advantage?}

The direct-action advantage in this study is consistent with the idea that the dorsal stream can guide visual actions with a higher degree of spatial resolution than is available to the ventral stream. Yet, we acknowledge that this finding is far from intuitive when considered from the perspective of conventional theories of visual search. Consider the task demands involved in making each of the two types of localization response. In both versions, the observer's motor response that was ultimately recorded was a keypress that was spatially mapped to the viewing screen. Also, in both versions, the observers had to note the letter label of the screen grid location so that they could press the corresponding key. The only difference between the two response sequences was that in the directaction condition, the observers were required to first point to the screen before pressing the key with the same finger. From the perspective of a unitary information-flow model, all the information needed to make the correct keypress was available at the eye in an equivalent way for both conditions. This information should therefore have been equally available to the keypressing finger. The extra step of pointing to the screen might even have interfered with accurate responding, because of the additional delay or the added cognitive requirements of programming and executing the pointing response. Yet, the results showed that performance of this additional step led to more accurate target localization. The dual systems theory suggests that this is because the pointing response capitalized on the dorsal visual stream, which is designed to process spatial locations with metric precision. When the observer pointed directly to the target, the benefits of this spatial precision could be harnessed in the response.

The direct action in this study involved pointing to the target location after the search display had been replaced with the response grid. One premise of the dual systems 
theory is that dorsal stream processing is online and short-lived, enabling it to respond appropriately to objects in a dynamic way. As the delay increases between visual input and response initiation, motor responses switch their reliance from dorsal stream to ventral stream information. Empirical estimates of position memory in the dorsal stream are less than $2 \mathrm{sec}$ in some studies (Elliott \& Madalena, 1987; Goodale, Jakobson, \& Keillor, 1994; Westwood, Heath, \& Roy, 2000, 2001) and up to 2 min in others (e.g., Creem \& Proffitt, 1998). In the present study, the observers were allowed to initiate localization immediately following target offset. The greater accuracy found for direct action suggests that dorsal stream information was still available to guide this response.

In addition to the higher spatial resolution of the dorsal stream, there are several other mechanisms that could contribute to the direct-action advantage and that should therefore be considered in future studies. One possibility is that direct and indirect actions are each associated with different eye movement patterns. Different eye movements could be involved for simple pragmatic reasons (e.g., precise eye movement may be required to guide the hand to a specific display location) as well as for reasons having to do with dorsal-ventral specialization. For example, it has been proposed that object locations are referenced in the dorsal stream with regard to the viewer's egocenter. This means that accurate reaching toward an object involves transforming the retinal coordinates of target location to limb-centered coordinates (Jeannerod, 1988), a transformation that seems to involve the posterior parietal cortex (Buneo, Jarvis, Batista, \& Andersen, 2002). For an indirect action, coding of object location is thought to be done in an exocentric or world-based frame of reference. Thus, when an object-directed action is not required, less accurate spatial location information will be preserved, simply because no direct limb movement is required. The relative positions in the display can simply be mapped to the relative positions on the response grid, with no need for the additional precision required for real-world interaction. These differences in spatial referencing may be evident in detailed eye movement records of observers performing direct and indirect target localization.

A second possibility is that movement intention or preparation has different influences on direct and on indirect actions. Premotor theory postulates that spatial attention is a consequence of a prepared but nonexecuted oculomotor program (Rizzolatti, Riggio, Dascola, \& Umiltà, 1987; Rizzolatti, Riggio, \& Sheliga, 1994). In an application of this theory to visually guided grasping, it was shown that preparing to grasp an obliquely oriented bar facilitated response times to a similar visual stimulus. This facilitation occurred even when the prepared grasp was inhibited and a different motor response, such as a footpress or an eyeblink, was required (Craighero, Fadiga, Rizzolatti, \& Umiltà, 1999). This suggests that motor preparation has facilitatory effects that remain even when the prepared movement is inhib- ited and another movement is required. In the present context, this implies that the motor preparation involved in direct pointing may be the sole critical ingredient in the direct-action advantage. In a future study, this hypothesis could be tested by requiring a direct-action response on most trials but including trials in which only an indirect action is required. If preparation alone is sufficient, then the direct-action advantage should be observed even on these overtly indirect responses.

\section{Implications for Visual Search Involving Target Detection Versus Target Localization}

Target localization accuracy in the present study was generally quite high for both direct and indirect actions, regardless of whether target detection was required first or second. This high degree of spatial resolution for localization is compatible with both feature integration theory and texton theory. From the perspective of feature integration theory (Treisman \& Gelade, 1980; Treisman $\&$ Sato, 1990), many of the present targets were difficult to detect, meaning that attention was necessarily focused on specific locations. This result is also not surprising according to texton theory (Julesz, 1984; Sagi \& Julesz, 1985 a), because localization is generally thought to occur prior to detection.

However, neither of these theories makes a prediction with respect to the superiority of direct over indirect actions for target localization. This is because action and preparation for action play no role in either of these theories. As is true for most theories prior to dual systems theory, visual perception is thought to be complete prior to the processes that are relevant for response planning and execution.

A question remains as to how traditional theories of visual search might be accommodated with respect to dual systems theory. It is possible that the situations in which feature integration and texton theory apply are strictly limited to ventral stream processing, particularly given that they are built on experimental paradigms that require conscious reports and do not involve visually guided actions. In previous studies in this vein, localization responses that depend on putative ventral stream functions, such as written responses (Treisman \& Gelade, 1980) and arbitrarily mapped keypresses and switches (e.g., Green, 1992), have been used.

\section{Implications for Dual-Task Performance}

Because the ventral and dorsal systems can function independently to some extent, it is possible that each system has its own pool of attentional resources. The present finding that direct target localization is relatively unaffected by being preceded by target detection suggests that direct action does not rely on the same resources as target detection. This is in contrast with indirect target localization, which is disrupted when preceded by target detection.

There are at least two ways to account for this result. One possibility is that the two streams operate so independently that dorsal and ventral tasks can be performed con- 
currently without interference. The present results are consistent with this idea. A way of refining this idea in future research would be to systematically manipulate the extent to which detection and localization depended on the same system. For example, if the detection and localization responses both depended on visually guided pointing (e.g., pointing to a sample of the seen target below the display before pointing to its screen location), there should be greater dual-task costs than those found in the present study. At the same time, making target localization rely even less on the dorsal stream (e.g., by requiring a vocal response) should also increase the dual-task costs of detection and localization. Insofar as each task is dependent on a separate stream, there should be no dual-task costs.

A second possibility is that the task completed without interference (direct localization) is simply easier and less attentionally demanding overall. As such, it did not experience interference from the concurrent detection task, because it required very few resources. We think this option is unlikely because, in the way that the directaction response was implemented in our study, it required all of the same steps as the indirect action and even one extra step (i.e., pointing to the screen). On this account, adding the step of overt pointing must have somehow simplified the mental operations involved, even to the point of removing the need for attention. Far-fetched as that may seem, future studies will be able to determine the attention load associated with direct-action tasks by combining them with a variety of other dorsal and ventral stream tasks. It may turn out that dorsal stream tasks tend to compete with one another for the same cognitive resources but not with ventral stream tasks, or even that dorsal stream tasks simply do not draw on attentional resources in the conventional sense usually associated with conscious vision.

\section{Implications for Everyday Action}

The advantage found in this study for direct target localization points to the importance of taking the action of the observer into account in visual search. This should serve as a reminder that the contents of an observer's visual experience are not independent of his or her goals and expectations. For instance, in the realm of humanmachine interfaces, the use of a touch screen may contribute to more accurate perceptions and more accurate visually guided responses. It may even do so with a smaller cognitive load than would be expected if responses were made through a more abstract spatial mapping. Given the present findings, it will be important in future research to examine more closely the relationship between the accuracy of visual search and the response mode. The larger lesson is that whenever the observer is confronted with dual tasks, it is important to try to combine tasks that share as few cognitive resources with each other as possible. The distinction between human vision for perception versus vision for action seems to be a natural division, one that could be exploited effectively in the design of many human-machine interfaces.

\section{REFERENCES}

Aglioti, S., DeSouza, J. F. X., \& Goodale, M. A. (1995). Size-contrast illusions deceive the eye but not the hand. Current Biology, 5, 679685 .

Aks, D., \& ENNs, J. T. (1996). Visual search for size is influenced by a background texture gradient. Journal of Experimental Psychology: Human Perception \& Performance, 22, 1467-1481.

AtKinson, J., \& BRADDICK, O. J. (1989). "Where" and "what" in visual search. Perception, 18, 181-189.

BÁLINT, R. (1909). Seelenlähmung des Schauens, optische Ataxie, räumliche Störung der Aufmerksamkeit. Monatzeitschrift für Psychiatrie und Neurologie, 25, 51-81.

Braun, J., \& SAGI, D. (1990). Vision outside the focus of attention. Perception \& Psychophysics, 48, 45-58.

BRAUn, J., \& SAGI, D. (1991). Texture-based tasks are little affected by second tasks requiring peripheral or central attentive fixation. Perception, 20, 483-500.

Brenner, E., \& SMeEts, J. B. J. (1996). Size illusion influences how we lift but not how we grasp an object. Experimental Brain Research, 111, 473-476.

Buneo, C. A., Jarvis, M. R., Batista, A. P., \& Andersen, R. A. (2002). Direct visuomotor transformations for reaching. Nature, 416 632-636.

Castiello, U., Paulignan, Y., \& Jeannerod, M. (1991). Temporal dissociation of motor responses and subjective awareness. Brain, 114, 2639-2655

Craighero, L., Fadiga, L., Rizzolatti, G., \& Umiltà, C. (1999). Action for perception: A motor-visual attentional effect. Journal of Experimental Psychology: Human Perception \& Performance, 25, 1673-1692.

Creem, S. H., \& Proffitt, D. R. (1998). Two memories for geographical slant: Separation and interdependence of action and awareness. Psychonomic Bulletin \& Review, 5, 22-36.

Di Lollo, D., Kawahara, J., Zuvic, S. M., \& Visser, T. A. W. (2001). The preattentive emperor has no clothes: A dynamic redressing. Journal of Experimental Psychology: General, 130, 479-492.

Elliott, D., \& Madalena, J. (1987). The influence of premovement visual information on manual aiming. Quarterly Journal of Experimental Psychology, 39A, 541-559.

ENNS, J. T., \& RENSINK, R. A. (1990a). Influence of scene-based properties on visual search. Science, 247, 721-723.

Enns, J. T., \& Rensink, R. A. (1990b). Sensitivity to three-dimensional orientation in visual search. Psychological Science, 1, 323-326.

Enns, J. T., \& Rensink, R. A. (1991). VSearch Color: Full-color visual search experiments on the Macintosh II. Behavior Research Methods, Instruments, \& Computers, 23, 265-272.

Fecteau, J. H., Chua, R., Franks, I., \& Enns, J. T. (2001). Visual awareness and the on-line modification of action. Canadian Journal of Experimental Psychology, 55, 106-112.

Goodale, M. A., Jakobson, L. S., \& Keillor, J. M. (1994). Differences in the visual control of pantomimed and natural grasping movements. Neuropsychologia, 32, 1159-1178.

Goodale, M. A., \& Milner, A. D. (1992). Separate visual pathways for perception and action. Trends in Neurosciences, 15, 20-25.

Goodale, M. A., Milner, A. D., Jakobson, L. S., \& Carey, D. P. (1991). A neurological dissociation between perceiving objects and grasping them. Nature, 349, 154-156.

Goodale, M. A., Pélisson, D., \& Prablanc, C. (1986). Large adjustments in visually guided reaching do not depend on vision of the hand or perception of target displacement. Nature, 320, 748-750.

Green, M. (1992). Visual search: Detection, identification, and localization. Perception, 21, 765-777.

Haxby, J. V., Grady, C. L., Horwitz, B., Ungerleider, L. G. Mishin, M., Carson, R. E., Herscovitch, P., Schapiro, M. B., \& RAPOPORT, S. I. (1991). Dissociation of object and spatial visual processing pathways in human extrastriate cortex. Proceedings of the National Academy of Sciences, 88, 1621-1625.

HeLd, R. (1970). Two modes of processing spatially distributed visual stimuli. In F. O. Schmitt (Ed.), The neurosciences: Second study program (pp. 317-324). New York: Rockefeller University Press. 
Ho, C. E. (1998). Letter recognition reveals pathways of second-order and third-order motion. Proceedings of the National Academy of Sciences, 95, 400-404.

JaCkson, S. R. \& Shaw, A. (2000). The Ponzo illusion affects gripforce but not grip-aperture scaling during prehension movements. Journal of Experimental Psychology: Human Perception \& Performance, 26, 418-423.

JEANnerod, M. (1988). The neural and behavioral organization of goal-directed movements. New York: Oxford University Press.

Joseph, J. S., Chun, M. M., \& Nakayama, K. (1997). Attentional requirements in a "preattentive" feature search task. Nature, 387, 805807.

JuLESZ, B. (1984). A brief outline of the texton theory of human vision. Trends in Neuroscience, 7, 41-45.

Milner, A. D., \& Goodale, M. A. (1995). The visual brain in action. Oxford: Oxford University Press.

Milner, A. D., Perrett, D. I., Johnston, R. S., Benson, P. J., Jordan, T. R., Heeley, D. W., Bettucci, D., Mortara, F., Mutani, R., Terazzi, E., \& Davidson, D. L. W. (1991). Perception and action in "visual form agnosia." Brain, 114, 405-428.

Navon, D., \& Gopher, D. (1979). On the economy of the humanprocessing system. Psychological Review, 86, 214-255.

Norman, D. A., \& Bobrow, D. G. (1975). On data-limited and resourcelimited processes. Cognitive Psychology, 7, 44-64.

Norman, D. A., \& Bobrow, D. G. (1976). On the analysis of performance operating characteristics. Psychological Review, 83, 508-510.

Norman, J. (2002). Two visual systems and two theories of perception: An attempt to reconcile the constructivist and ecological approaches. Behavioral \& Brain Sciences, 25, 73-144.

Perenin, M.-T., \& Vighetto, A. (1988). Optic ataxia: A specific disruption in visuomotor mechanisms. I. Different aspects of the deficit in reaching for objects. Brain, 111, 643-674.

Rizzolatti, G., Riggio, L., Dascola, I., \& Umiltà, C. (1987). Reorienting attention across the horizontal and vertical meridians: Evidence in favor of a premotor theory of attention. Neuropsychologia, 25, 31-40.

Rizzolatti, G., Riggio, L., \& Sheliga, B. M. (1994). Space and selective attention. In C. Umiltà \& M. Moscovitch (Eds.), Attention and performance $X V$ : Conscious and nonconscious information processing (pp. 231-265). Cambridge, MA: MIT Press, Bradford Books.

SAGI, D., \& Julesz, B. (1985a). Detection versus discrimination of visual orientation. Perception, 14, 619-628.

SAGI, D., \& Julesz, B. (1985b). "What" and "where" in vision. Science, 228, 1217-1219.
Schneider, G. E. (1969). Two visual systems. Science, 163, 895-902. Stevens, K. A. (1983). Slant-tilt: The visual encoding of surface orientation. Biological Cybernetics, 46, 183-195.

Tootell, R. B. H., Dale, A. M., Sereno, M. I., \& Malach, R. (1996). New images from human visual cortex. Trends in Neurosciences, 19 481-489.

Treisman, A., \& Gelade, G. (1980). A feature-integration theory of attention. Cognitive Psychology, 12, 97-136.

Treisman, A., \& Gormican, S. (1988). Feature analysis in early vision: Evidence from search asymmetries. Psychological Review, 95, 15 48.

Treisman, A., \& Sato, S. (1990). Conjunction search revisited. Journal of Experimental Psychology: Human Perception \& Performance, 16, 459-478.

Trevarthen, C. B. (1968). Two mechanisms of vision in primates. Psychologische Forschung, 31, 299-337.

Ungerleider,L. G., \& HaXby, J. V. (1994). "What" and "where" in the human brain. Current Opinion in Neurobiology, 4, 157-165.

Ungerleider, L. G., \& Mishin, M. (1982). Two cortical visual systems. In D. J. Ingle, M. A. Goodale, \& R. J. W. Mansfield (Eds.), Analysis of visual behavior (pp. 549-586). Cambridge, MA: MIT Press.

VAn Essen, D. C., \& DeYoe, E. A. (1995). Concurrent processing in the primate visual cortex. In M. S. Gazzaniga (Ed.), The cognitive neurosciences (pp. 383-400). Cambridge, MA: MIT Press.

Westwood, D. A., Heath, M., \& Roy, E. A. (2000). The effect of a pictorial illusion on closed-loop and open-loop prehension. Experimental Brain Research, 134, 456-463.

Westwood, D. A., Heath, M., \& Roy, E. A. (2001). The accuracy of reaching movements in brief delay conditions. Canadian Journal of Experimental Psychology, 55, 304-310.

Wickens, C. D. (1984). Processing resources in attention. In R. Parasuraman \& D. R. Davies (Eds.), Varieties of attention (pp. 63-101). Orlando, FL: Academic Press.

Wolfe, J. M., Friedman-Hill, S. R., Stewart, M. I., \& O'Connell, K. M. (1992). The role of categorization in visual search for orientation. Journal of Experimental Psychology: Human Perception \& Performance, 18, 34-49.

(Manuscript received April 30, 2002; revision accepted for publication January 2, 2003.) 